\title{
DR-27. NANODEFECTS IN HIGHLY IMPERFECT OPTICAL CRYSTALS
}

\author{
V.M. Lisitsyn $^{1}$, L. A. Lisitsyna ${ }^{2}$, E. F. Polisadova ${ }^{1}$, A. T. Tulegenova ${ }^{1}$ \\ ${ }^{1}$ National Research Tomsk Polytechnic University, Lenin Av., 30, Tomsk, 634050, Russia \\ ${ }^{2}$ Tomsk State University of Architecture and Building, Soljanoj St., 2, Tomsk, 634003, Russia \\ E-mail: lisitsyn@tpu.ru
}

We have studied general characteristics of luminescence in the tungsten doped lithium fluoride crystal, magnesium fluoride ceramics, and zinc tungstate crystal. The comparison of the luminescence spectra, excitation, absorption and kinetic characteristics of the luminescence decay revealed a number of common features. It is concluded that nanodefects of similar composition, spatial and energy structure enter these materials during synthesis. We proposed a band scheme of the system, which is embedded in the matrix band structure of the nanodefect phase with radiative levels of the luminescence centers, the structural elements of nanodefects.

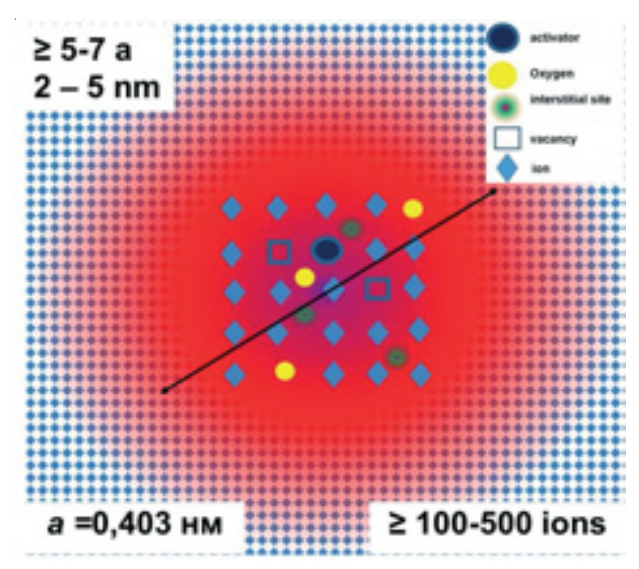

$a$

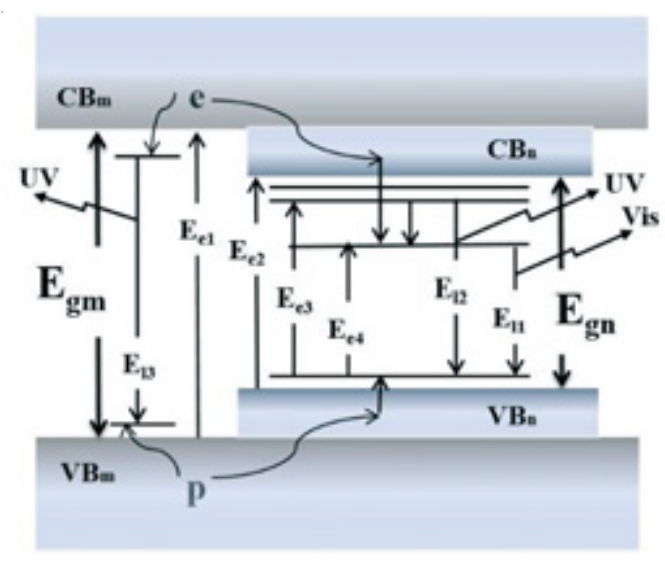

$b$

The expected spatial structure of the nanodefect in doped LiF crystal $(a)$ and band scheme for crystal with nanodefects $(b)$

Experiments were carried out within the framework of the development program of the National Research Tomsk Polytechnic University in the project of the leading universities of theworld 5-100 (Project № VIU-OM-205/2018). 